\title{
Oxiuriasis apendicular: estudio de prevalencia y descripción clínico-morfológica*
}

\author{
Drs. ÓSCAR TAPIA E. ${ }^{1,2}$, CÉSAR MUÑOZ C. ${ }^{3}$ \\ 1 Departamento de Anatomía Patológica. Facultad de Medicina. Universidad de La Frontera. \\ 2 BIOREN (Scientifical and Technological Bioresources Nucleus). \\ 3 Departamento de Cirugía y Traumatología, Facultad de Medicina. Universidad de La Frontera. \\ Temuco, Chile.
}

\begin{abstract}
\section{Presence of Enterobius vermicularis in surgical pieces of appendectomies}

Background: The frequency of appendicular presence of Enterobius vermicularis varies from 0.2 to $41.8 \%$. It is generally a pathological finding. The luminal obstruction by the parasite may unveil an acute appendicitis. Aim: To study the frequency of Enterobius vermicularis presence in surgical pieces of appendectomies. Material and Methods: Analysis of surgical pieces of appendectomies processed at the pathology laboratory of a general hospital, between 1993 and 2010. Results: In 21.038 surgical pieces, the presence of Enterobius vermicularis was found in $322(1.5 \%)$. Fifty nine percent were women and $71 \%$ were younger than 18 years. Acute inflammation was confirmed in $71 \%$. A history of previous abdominal pain was present in 40 and $70 \%$ of patients with and without acute inflammation in the surgical piece. Conclusions: In this series, the frequency of Enterobius vermicularis presence in surgical pieces of appendectomy was $1.5 \%$.
\end{abstract}

Key words: Enterobius vermicularis, acute appendicitis, appendectomy.

\section{Resumen}

Introducción: La frecuencia de Enterobius vermicularis (EB) apendicular varía entre 0,2-41,8\%, siendo generalmente su diagnóstico un hallazgo al estudio histopatológico. La obstrución luminal puede desencadenar un cólico apendicular o evolucionar a una apendicitis aguda, siendo por tanto una causa frecuente de apendicectomía. El objetivo del estudio es determinar la prevalencia de EB en piezas quirúrgicas de apendicectomía junto con describir características clínico-morfológicas. Material y Método: Cohorte retrospectiva de pacientes con oxiuriasis apendicular diagnosticados en el Hospital Hernán Henríquez Aravena entre 1993-2010. Se estudiaron variables clínico-morfológicas, utilizándose estadística descriptiva y analítica, aplicando Chicuadrado y test exacto de Fisher para variables categóricas y T-student para variables continuas con un IC de 95\%. Resultados: En 21.038 apendicectomías se verificaron EB en 322 casos (1,53\%). El 59\% eran mujeres y $71 \%$ menor de 18 años. Inflamación aguda fue confirmada en $71 \%$. En casos con inflamación aguda la temperatura rectal y recuento leucocitario promedio fue $38^{\circ} \mathrm{C}$ y $12.975 \mathrm{~mm}^{3}$ respectivamente, mientras que en casos sin inflamación $37,8^{\circ} \mathrm{C}$ y $10.984 \mathrm{~mm}^{3}(\mathrm{p}=\mathrm{N} . \mathrm{S})$. En el grupo apendicectomizado por sospecha de apendicitis el $40 \%$ tenía historia de dolor abdominal previo, mientras que en aquellos sin inflamación aguda asociada el

*Recibido el 22 de mayo de 2011 y aceptado para publicación el 19 de junio de 2011.

Correspondencia: Dr. Oscar Tapia E.

Manuel Montt 112. Código Postal 478-1176. Temuco, Chile.

otescalona@gmail.com 
$72 \%(p=0,005)$. Conclusiones: La prevalencia de EB apendicular fue 1,53\%, afectando fundamentalmente a niños-jóvenes del sexo femenino. La oxiuriasis apendicular representa una causa de dolor abdominal interpretado como apendicitis aguda, sin asociarse a inflamación en el examen anatomopatológico. La historia de dolor abdominal previo resultó una variable útil para discriminar cólico apendicular de apendicitis aguda.

Palabras clave: Apéndice, Enterobius vermicularis, oxiuriasis.

\section{Introducción}

La apendicectomía por apendicitis aguda representa la causa más frecuente de cirugía en los servicios de urgencia en el mundo, siendo la probabilidad de ser sometido a esta cirugía en la vida de un $12 \%$ y $25 \%$ para hombres y mujeres respectivamente ${ }^{1-4}$.

El examen histopatológico de la pieza operatoria permite en gran parte de los casos confirmar el diagnóstico clínico de apendicitis; reportándose, sin embargo, hasta en un 15\% la ausencia de inflamación al estudio anatomopatológico de las piezas quirúrgicas ${ }^{3}$. Junto con demostrar la presencia o no de inflamación, el estudio histopatológico permite la pesquisa de lesiones neoplásicas apendiculares generalmente inaparentes al examen clínico e imagenológico, siendo su frecuencia reportada en hasta un $0,5 \%$ de las apendicectomías ${ }^{5-8}$; del mismo modo y en un porcentaje variable permite identificar elementos parasitarios (Enterobius vermicularis, Entamoeba histolytica, Ascaris lumbricoides, Trichuris trichiura, Schistosoma spp, Taenia spp), asociados o no a inflamación apendicular ${ }^{1,9-12}$.

En cuanto a la presencia de elementos parasitarios en el lumen apendicular estos pueden obstruirlo y provocar lo que se denomina "cólico apendicular", sin desencadenar necesariamente una respuesta inflamatoria, hecho que explica que parte de los apéndices cecales examinados en los que se evidencian parásitos no presenten inflamación aguda asociada, siendo por tanto clínicamente una causa frecuente de casos falsos positivos de apendicitis aguda en pacientes sometidos a cirugía; sin embargo, en caso de prolongarse la obstrucción luminal pueden favorecer el sobrecrecimiento bacteriano activando finalmente la cascada de la respuesta inflamatoria ${ }^{1,13-16}$. En estos casos el diagnóstico e identificación del parásito está dado por el examen histopatológico; siendo la infección por Enterobius vermicularis (EB) la más frecuente descrita a nivel apendicular ${ }^{9,17}$. Este parásito es un nemátodo, donde el hombre es el único huésped natural conocido, no requiriendo de un huésped intermedio, ni prolongada incubación exógena para completar su ciclo vital pudiendo transmitirse mediante la ingestión de huevos a través de las manos contaminadas por el contacto con la región perianal, contaminación de alimentos y/o del agua y la inhalación o deglución de huevos directamente desde el ambiente?
El objetivo del presente estudio es determinar la prevalencia de Enterobius vermicularis en piezas quirúrgicas de apendicectomía, junto con describir las características clínico-morfológicas de este grupo de sujetos.

\section{Material y Método}

\section{Diseño del estudio \\ Cohorte retrospectiva.}

\section{Criterios de inclusión}

Se incluyeron todos los casos de pacientes sometidos a apendicectomía y cuyas muestras fueron examinadas en la Unidad de Anatomía Patológica del Hospital Dr. Hernán Henríquez Aravena de Temuco, desde enero de 1993 hasta diciembre de 2010 $(\mathrm{n}=21.038)$ y cuyo diagnóstico incluía la presencia de EB $(n=322)$. No se consideraron criterios de exclusión.

\section{Protocolo de procesamiento}

En este período, el procesamiento de las piezas quirúrgicas de apendicectomía fue realizado mediante sección longitudinal de la pieza operatoria, tomándose en todas ellas muestras del tercio distal, considerando para apéndices macroscópicamente sanos el estudio completo de la pieza quirúrgica y/o toma de muestras adicionales en caso de existir lesiones neoplásicas sospechosas al examen macroscópico.

\section{Variables estudiadas}

En los casos con diagnóstico de oxiuriasis apendicular se estudiaron las siguientes variables: Edad, género, diagnóstico clínico, historia previa de dolor abdominal según registro en ficha clínica o atenciones de urgencia caracterizado por dolor abdominal localizado preferentemente en cuadrante inferior derecho, temperatura corporal a nivel rectal, recuento de leucocitos pre-operatorio, existencia de eosinofilia en el hemograma (definido como recuento de eosinófilos superior a 500 por $\mathrm{mm}^{3}$ ) y presencia o no de inflamación aguda apendicular asociada.

\section{Plan de análisis}

Utilizando los paquetes estadísticos Epi-info 6.0 y Stata 9.0, se realizó un análisis exploratorio 
de los datos y posteriormente se aplicó estadística descriptiva con cálculo de promedios y desviaciones estándar, medianas y valores extremos para variables continuas; y cálculo de porcentajes para variables categóricas. Luego, se aplicó estadística analítica, utilizando t-test y análisis de la varianza (ANOVA) para variables continuas, $\chi^{2}$ de Pearson y exacto de Fisher para variables categóricas.

\section{Resultados}

Se realizaron en el período analizado 21.038 apendicetomías, diagnosticándose en 322 casos $(1,53 \%)$ la presencia de EB.

El $59 \%$ de los sujetos en estudio eran mujeres. La edad promedio del grupo fue $18 \pm 16,8$ años (rango: 1-88 años), correspondiendo el $71 \%$ a pacientes menores de 18 años.

En el 89\% (287) de los casos el diagnóstico clínico pre-operatorio que justificó la cirugía fue un cuadro clínico sugerente de apendicitis, mientras que en el 11\% (35) la apendicectomía se realizó en cirugías abdominales por otras causas (patología ginecológica [absceso tubo-ovárico 6 , torsión quiste anexial 3], patología gastro-intestinal [divertículo de Meckel 4, hernia umbilical e inguinal 2, hidatidosis hepática 4, colelitiasis 14], patología nefro-urológica [absceso peri-renal 1, cáncer de testículo 1]), de acuerdo al protocolo operatorio se evidenció durante la cirugía la presencia de elementos parasitarios en el ostium apendicular en tan sólo 5 casos operados por sospecha de apendicitis aguda (Figura 1).

En los casos con cuadro clínico sugerente de apendicitis aguda el proceso inflamatorio agudo fue confirmado histológicamente en $71 \%$ (204) de los pacientes operados que conjuntamente presentaban EB en el lumen apendicular (Figura 2).

En sujetos apendicectomizados por sospecha clínica de apendicitis aguda la totalidad de pacientes presentó dolor abdominal localizado en fosa ilíaca derecha, siendo la temperatura rectal promedio $37,9^{\circ} \mathrm{C}\left(37,5^{\circ} \mathrm{C}-38,6^{\circ} \mathrm{C}\right)$ y una mediana de $38^{\circ} \mathrm{C}$. $\mathrm{El}$ recuento leucocitario promedio fue de 12.457 $\mathrm{mm}^{3}$ (9.721 $\left.\mathrm{mm}^{3}-15.874 \mathrm{~mm}^{3}\right)$; presentando eosinofilia en el hemograma el 6\%, mientras que en aquellos pacientes apendicectomizados por otras causas tan sólo 1 sujeto $(1 \%)(\mathrm{p}=0,08)$.

Para los casos con inflamación aguda confirmada histológicamente el promedio de la temperatura rectal y recuento leucocitario fue $38{ }^{\circ} \mathrm{C}\left(37,8{ }^{\circ} \mathrm{C}\right.$ $\left.38,6{ }^{\circ} \mathrm{C}\right)$ y $13.930 \mathrm{~mm}^{3}\left(11.542 \mathrm{~mm}^{3}-15.874\right.$ $\mathrm{mm}^{3}$ ); mientras que para casos sin inflamación aguda asociada fueron $37,8^{\circ} \mathrm{C}\left(37,5^{\circ} \mathrm{C}-38,4^{\circ} \mathrm{C}\right) \mathrm{y}$ $10.984 \mathrm{~mm}^{3}\left(9.721 \mathrm{~mm}^{3}-12.115 \mathrm{~mm}^{3}\right)(\mathrm{p}=\mathrm{N} . \mathrm{S})$.

De la totalidad del grupo estudiado, el 54\% (175) registraba en su ficha clínica consultas previas por dolor abdominal. Del grupo apendicectomizado por sospecha de apendicitis aguda con confirmación histológica el 40\% (81) había consultado por dolor abdominal a lo menos en una oportunidad en los 2 años previos a la cirugía, mientras que en aquellos sin inflamación aguda asociada el 57\% (48) presentaba consultas previas $(\mathrm{p}=0,005)$.

Tabla 1. Distribución de variables clínico-morfológicas en apendicectomías de urgencia por sospecha de apendicitis aguda

\begin{tabular}{|c|c|c|c|}
\hline & $\begin{array}{l}\text { Sin inflamación aguda } \\
\qquad(\mathrm{n}=\mathbf{8 3})\end{array}$ & $\begin{array}{l}\text { Con inflamación aguda } \\
\qquad(n=204)\end{array}$ & $\mathbf{p}$ \\
\hline $\begin{array}{l}\text { Género } \\
\text { Femenino } \\
\text { Masculino }\end{array}$ & $\begin{array}{l}58 \%(48) \\
42 \%(35)\end{array}$ & $\begin{array}{l}61 \%(124) \\
39 \%(80)\end{array}$ & 0,64 \\
\hline Edad (Promedio) & $15,7 \pm 15$ años & $17,2 \pm 13$ años & 0,57 \\
\hline Temperatura rectal (Promedio) & $37,8^{\circ} \mathrm{C}$ & $38,0^{\circ} \mathrm{C}$ & 0,75 \\
\hline Recuento leucocitario (Promedio) & $10.984 \mathrm{~mm}^{3}$ & $13.930 \mathrm{~mm}^{3}$ & 0,18 \\
\hline $\begin{array}{l}\text { Eosinofilia plasmática } \\
\text { No } \\
\text { Sí }\end{array}$ & $\begin{array}{l}99 \%(82) \\
1 \%(1)\end{array}$ & $\begin{array}{l}94 \%(192) \\
6 \%(12)\end{array}$ & 0,08 \\
\hline $\begin{array}{l}\text { Historia de dolor abdominal } \\
\text { No } \\
\text { Sí }\end{array}$ & $\begin{array}{l}43 \%(35) \\
57 \%(48)\end{array}$ & $\begin{array}{l}60 \%(123) \\
40 \%(81)\end{array}$ & 0,005 \\
\hline
\end{tabular}




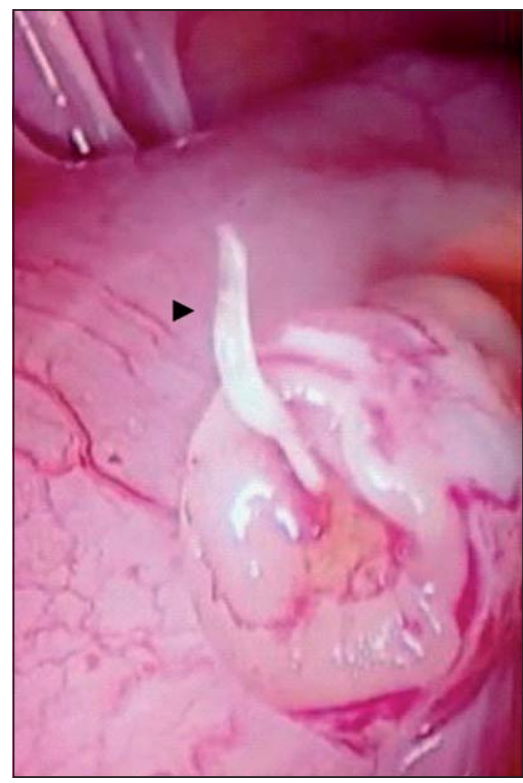

Figura 1. Se aprecia zona cecal saliendo a través del ostium del muñón apendicular estructura aplanada y alargada, blanquecina compatible con forma adulta de Enterobius vermicularis ( $)$.

\section{Discusión}

La infección gastro-intestinal por EB presenta una distribución universal y es considerada la infección helmíntica más frecuente, presentándose en todas las edades y niveles socio-económicos, predominando, sin embargo, en niños y jóvenes; estimándose en alrededor de 1.000 millones las personas afectadas en el mundo ${ }^{1,9,18}$. En la mayoría de las personas esta infección es asintomática y su manifestación clínica característica representada por prurito anal, esto explicado por el ciclo biológico del parásito, en el cual las hembras maduras y fecundadas migran desde la zona ceco-apendicular hasta el margen anal para realizar la oviposición ${ }^{9}$.

Durante su recorrido por el tracto gastro-intestinal, el parásito puede localizarse en forma inhabitual en el apéndice cecal, siendo en 1899 Still, quien describe por primera vez el compromiso apendicular por EB, variando ampliamente la frecuencia reportada a nivel mundial, entre $0,2-41,8 \%$; en nuestra serie y al igual que en otros estudios la prevalencia alcanzó al 1,53\% afectando mayormente al grupo de niños y adultos jóvenes $(71 \%$ menores de 18 años) $)^{9,14,17,19,20}$.

La presencia de oxiuros en el apéndice cecal puede obstruir su lumen y provocar lo que se denomina "cólico apendicular", sin desencadenar necesariamente una inflamación apendicular ${ }^{1,13-16}$, hecho que explica la proporción de apéndices examinados en los cuales no se confirmó histológicamente la inflamación aguda (29\%).

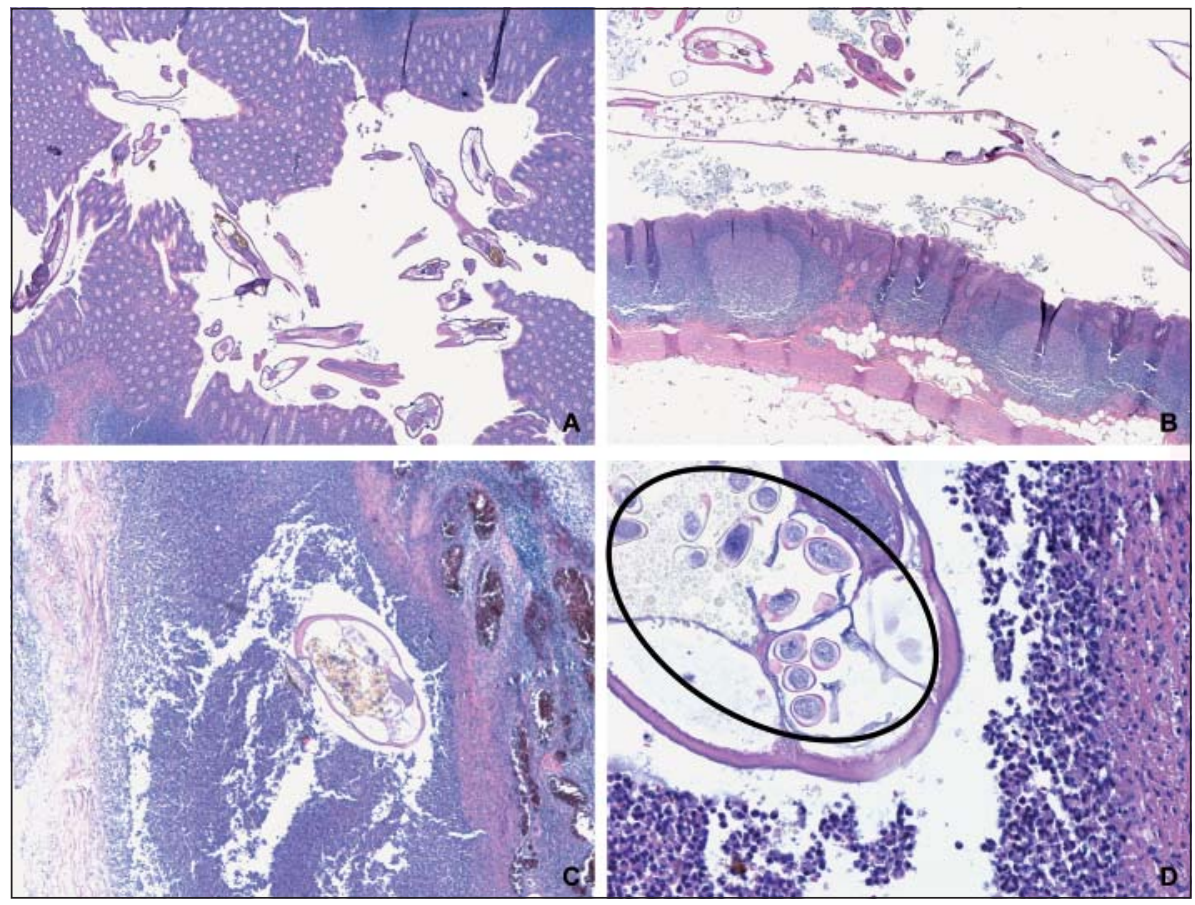

Figura 2. (H-E, A: 20x, B: 40x, C: 20x, D: 200x). A-B: Apéndice cecal con presencia en el lumen de fragmentos parasitarios correspondientes a Enterobius vermicularis en ausencia de infiltrado inflamatorio polimorfonuclear parietal. C-D: Apéndice cecal con proceso inflamatorio agudo de tipo flegmonoso con fragmentos parasitarios correspondientes a útero grávido de Enterobius vermicularis relleno de huevos (círculo). 
Sin embargo, esta obstrucción puede prolongarse y junto con provocar un cólico apendicular, favorecer el sobrecrecimiento bacteriano y finalmente ser el factor etiopatogénico de la apendicitis aguda como ocurrió en el $71 \%$ de los pacientes de la serie presentada, no observando diferencias estadísticamente significativas en grupos con y sin inflamación aguda en cuanto a la temperatura rectal ni recuento leucocitario; demostrándose a su vez tan sólo en un $6 \%$ de los pacientes aumento del recuento de eosinófilos en el hemograma, no siendo por tanto una herramienta útil en el diagnóstico de esta enfermedad.

Cabe mencionar que el dolor en fosa ilíaca derecha ocasionado por un cólico apendicular secundario a obstrucción luminal parasitaria puede ser indistinguible del de una apendicitis aguda, sin embargo, una exhaustiva historia y examen clínico, incluyendo observación clínica y posterior re-evaluación que demuestre franca disminución del dolor permitiría seleccionar casos compatibles con un cólico apendicular y, por lo tanto, evitar la cirugía y eventuales complicaciones operatorias ${ }^{1}$; en la cohorte estudiada el $40 \%$ de los casos operados con sospecha de apendicitis presentó historia de dolor abdominal previo mientras que el $57 \%$ de aquellos en los que no se demostró inflamación aguda al examen histopatológico había consultado previamente por dolor abdominal que posteriormente cedió, siendo por tanto este grupo susceptible de ser observado clínicamente y posteriormente re-evaluado.

Es importante mencionar que estos pacientes deben completar su tratamiento con medicamentos antihelmínticos benzoimidazólicos como el mebendazol o albendazol, de manera de tratar la enfermedad de base, teniendo en cuenta la frecuente reinfección y necesidad de tratamiento al círculo familiar cercano, incluso en aquellos asintomáticos que pudiesen servir de reservorio en caso de no ser tratados ${ }^{1,9}$.

\section{Referencias}

1. Aydin O. Incidental parasitic infestations in surgically removed appendices: a retrospective analysis. Diagnostic Pathology 2007;2:16-20.

2. Flum DR, Koepsell T. The clinical and economic correlates of misdiagnosed appendicitis. Arch Surg. 2002;137:799-804.

3. Shelton T, McKinlay R, Schwartz RW. Acute appendicitis current diagnosis and treatment. Curr Surg. 2003;60:502-5.

4. Ariyarathenam AV, Nachimuthu S, Tang TY, Courtney ED, Harris SA, Harris AM. Enterobius vermicularis infestation of the appendix and management at the time of laparoscopic appendectomy: case series and literature review. Int J Surg. 2010;8:466-9.

5. Connor S, Hanna G, Frizelle F. Appendiceal tumors: retrospective clinicopathologic analysis of appendix tumors from 7970 appendectomies. Dis Colon Rectum 1998;41:75-80.

6. Graham RP, Williams NP, West KA. Primary epithelial tumours of the appendix in a black population: a review of cases. World J Gastroenterol. 2009;15:1472-4.

7. McGory M, Maggard M, Kang H, OConnell J, Ko C. Malignancies of the appendix: beyond case series reports. Dis Colon Rectum 2005;48:2264-71.

8. Tapia O, Manterola C, Villaseca M, Araya JC, Guzmán P, Roa JC. Descripción clínico-morfológica y factores pronósticos en carcinomas de apéndice cecal: Estudio de cohorte. Rev Chil Cir. 2010;62:255-61.

9. Cook GC. Enterobius vermicularis infection. Gut 1994;35:1159-62.

10. Gupta SC, Gupta AK, Keswani NK, Singh PA, Tripathi AK, Krishna V. Pathology of tropical appendicitis. J Clin Pathol. 1989;42:1169-72.

11. Lamps LW. Appendicitis and infections of the appendix. Semin Diagn Pathol. 2004;21:86-97.

12. Sartorelli AC, da Silva MG, Rodrigues MA, da Silva RJ. Appendiceal taeniasis presenting like acute appendicitis. Parasitol Res. 2005;97:171-2.

13. Yildırım S, Nursal TZ, Tarım A, Kayaselcuk F, Noyan T. A rare cause of acute appendicitis: parasitic infection. Scand J Infect Dis. 2005;37:757-9.

14. Sah SP, Bhadani PP. Enterobius vermicularis causing symptoms of appendicitis in Nepal. Trop Doct. 2006;36:160-162.

15. Dahlstrom JE, Macarthur EB. Enterobius vermicularis: a possible cause symptoms resembling appendicitis. Aust N Z J Surg. 1994;64:692-4.

16. Budd JS, Armstrong C. Role of Enterobius vermicularis in the aetiology of appendicitis. Br J Surg. 1987;74:7489.

17. Marjorie JA, Robert LG, Jonathan IG, Sue H, Donna AC. Clinical manifestations of appendiceal pinworms in children: an institutional experience and a review of literature. Pediatr Surg Int. 2004;20:372-5.

18. David E. Intestinal Worms. In Sleisenger and Fortdtran's Gastrointestinal and liver disease. Volume 2. 8th edition. Edited by: Feldman M, Friedman L, Brandt L. Philadelphia: Elsevier Saunders;2006:2442-3.

19. Still GF. Oxyuriasis vermicularis in children. Br Med J. 1899;1:898-900.

20. de Silva DF, da Silva RJ, da Silva MG, Sartorelli AC, Rodrigues MA. Parasitic infection of the appendix as a cause of acute appendicitis. Parasitol Res. 2007;102:99102 . 